\title{
Expression of recombinant mouse cytosolic carboxypeptidase 6 in Escherichia coli
}

\author{
Ruixue Wang ${ }^{1, *}$, and Hui-Yuan $\mathrm{Wu}^{1, *}$ \\ ${ }^{1}$ School of Pharmaceutical Science and Technology, Tianjin University, Tianjin, 300072, China
}

\begin{abstract}
Cytosolic carboxypeptidase 6 (CCP6) is a member of cytosolic carboxypeptidase (CCP) family that catalyze the removal of polyglutamate side chains from protein substrates. Biochemical and biophysical characterization of CCPs requires large quantities of purified proteins. However, no method describing the expression of any mammalian CCP family member from bacteria has been published to our best knowledge. After considerable efforts to improve the solubility of mammalian CCPs expressed in bacteria, including the optimization of induction temperature and by using different receptive cells, we were able to get less expression of mouse CCP6 in soluble fraction of bacterial lysates. We report in this article, the bacterial expression of CCP6 using Arctic Express (DE3) competent cells that co-express the chaperonin system GroEL and GroES from Oleispira antarctica. However, to achieve a large number of soluble target proteins, the expression conditions still need to be further optimized.
\end{abstract}

\section{Introduction}

In mammals, cytosolic carboxypeptidase family catalyzes the demodification of a unique posttranslational modification-polyglutamylation. Polyglutamylation gives rise to variable lengths of glutamate side chains on the $\gamma$-carboxyl groups of glutamic acid residues in the gene-encoded sequence of the target proteins ${ }^{[1]}$. In the process of this modification, free glutamate residues are initially condensed to the $\gamma$-carboxyl group of a glutamate in the primary sequence of proteins to form a branched chain. Then additional glutamates are linked to this branching point glutamate by peptide bond ( $\alpha$-carboxyl) to form polyglutamylation side chains of different lengths (Figure 1). Its initiation and elongation are catalyzed by the tubulin tyrosine ligase like (TTLL) family [2, 3]. On the contrary, the six cytosolic carboxypeptidases catalyze the shortening and digestion of polyglutamylation chains ${ }^{[1,4-6]}$. CCP1 (also known as Nna1), CCP2, СCP3, СCP4 and СCP6 catalyze the removal of $\alpha$-carboxyl-linked glutamates. While CCP5 specifically removes the $\gamma$-carboxyl-linked, branching point glutamates ${ }^{[1]}$. This modification has been initially discovered on $\alpha$ - and $\beta$-tubulin ${ }^{[7,8]}$, which are the structural units of microtubules, and later also found on other proteins ${ }^{[9,10]}$.

Homologous CCP genes are widely distributed in different taxa from prokaryotes to eukaryotes. There are 1-2 genes of CCP in certain types of bacteria, and eukaryotic CCPs are widely distributed in holozoans and protists ${ }^{[1]}$. Unlike eukaryotic CCPs, which are involved in deglutamylation of tubulin and other proteins, the activity of bacterial CCPs has not been determined and their functions remain unknown. Currently, studying the biochemistry of eukaryotic CCPs depends on cell or insect cell expression systems, which are time-consuming and expensive. In fact, to date there are no published methods describing the expression and purification of any mammalian CCP family member from bacteria.

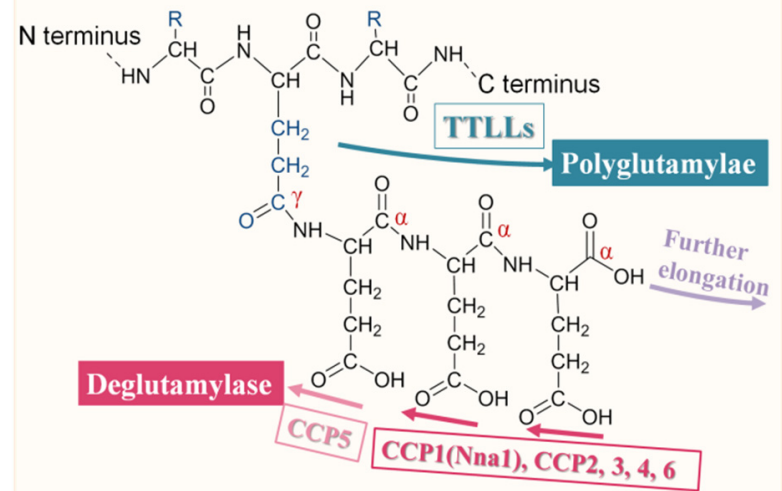

Fig. 1. A novel protein posttranslational modification Polyglutamylation. The formation of polyglutamate chains is catalyzed by the tubulin tyrosine ligase-like (TTLL) family. On

the contrary, the CCPs are responsible for metabolizing polyglutamate chains.

Although bacterial expression systems are cost-efficient and easy to be scaled up, protein insolubility often occurs when expressing eukaryotic proteins in a prokaryotic system. Optimization of expression conditions, such as induction temperature, the concentration of IPTG, and different bacterial strains, are often required to improve the solubility of target proteins. Here, using the smallest member of CCP family-CCP6 as a model for the CCP family, I systemically optimized the

\footnotetext{
*Corresponding author: 1336564890@qq.com; huiyuan.wu@tju.edu.cn
} 
expression condition of CCP6 in bacteria and a small amount of CCP6 expression in the soluble fractions of bacteria was achieved. Specifically, the CCP6 is expressed in the bacterial strain Arctic Express (DE3) that contains the chaperonins GroEL and GroES from a psychrophilic bacterium Oleispira antarctica. Expression along with this chaperonin system allows for the production of soluble CCP6 at low temperatures.

\section{Methods}

\subsection{Plasmid construction}

2 truncated variants of mouse CCP6 (FN429928): CCP6-45 (residues 45-440) and CCP6-167 (residues 167-439) were amplified by PCR and cloned into the pET 28a-HT vector at the SspI restriction site, using the Gibson Assembly® cloning protocol (New England Biolabs, Ipwich, MA, USA) ${ }^{[11]}$. The plasmids were extracted using the Plasmid DNA Purification Kit (OMEGA, USA) and sequenced by the company (Tsingke Biotech).

\subsection{Optimization of conditions for CCP6 expression in $E$. coli}

In order to obtain soluble target proteins, we optimized the induction temperature and time $\left(37^{\circ} \mathrm{C}\right.$ induction for 4 $\mathrm{h}, 30^{\circ} \mathrm{C}$ induction for $6 \mathrm{~h}, 16^{\circ} \mathrm{C}$ overnight induction), and used different host strains including Rosetta (DE3), and Arctic Express (DE3). A single colony was picked from the plate with toothpick and inoculated into $3 \mathrm{~mL} \mathrm{LB}$ liquid medium and cultured for 13 to 16 hours at $37^{\circ} \mathrm{C}$, $220 \mathrm{rpm}$. $1 \mathrm{~mL}$ of this culture was then inoculated to 100 $\mathrm{mL}$ fresh $\mathrm{LB}$ liquid medium, and cultured in the shaker at $37^{\circ} \mathrm{C}, 220 \mathrm{rpm}$ until the $\mathrm{OD}_{600}$ reached to $0.6-0.8$. Then CCP6 expression was induced with $0.4 \mathrm{mM}$ of IPTG and incubated at different temperature, $220 \mathrm{rpm}$. Next the cells were collected by centrifugation at $8,000 \mathrm{~g}$ for 10 min at $4^{\circ} \mathrm{C}$ and resuspended with $4 \mathrm{~mL}$ lysis buffer $(20$ $\mathrm{mM}$ HEPES, pH 7.4, $250 \mathrm{mM} \mathrm{KCl}, 25 \mu \mathrm{g} / \mathrm{mL}$ DNase, 25 $\mu \mathrm{g} / \mathrm{mL}$ Lysozyme, $10 \mathrm{mM}$ PMSF, $10 \mathrm{mM} \beta$-ME), then lysed by probe sonication for twice (each time $5 \mathrm{~min}, 50$ $\mathrm{W}, 1 \mathrm{~s}$ on/1 s off pattern). The supernatant and pellet were collected by centrifugation at $15,000 \mathrm{~g}$ for $30 \mathrm{~min}$ at $4^{\circ} \mathrm{C}$. The pellet was resuspended with $4 \mathrm{~mL}$ buffer $(20$ mM HEPES, $\mathrm{pH} 7.4,250 \mathrm{mM} \mathrm{KCl}) .80 \mu \mathrm{L}$ supernatant and pellet were taken out, added with $20 \mu \mathrm{L} 5 \mathrm{x}$ loading buffer followed by heating at $95^{\circ} \mathrm{C}$ for $10 \mathrm{~min}$. The expression level of CCP6 was confirmed via SDS-PAGE ( $10 \%$ polyacrylamide).

\subsection{Protein electrophoresis and immunoblotting}

Recombinant CCP6 were separated by $10 \%$ polyacrylamide hydrogel. After electrophoresis, proteins were transferred onto a nitrocellulose membrane using a Semi-dry transfer device (100 mA, $1 \mathrm{~h})$. Membranes were blocked with blocking buffer ( $5 \%$ milk/TBST), then incubated with mouse anti-His (1:5000; ImmunoWay,
USA) antibody overnight at $4^{\circ} \mathrm{C}$. After three times wash with TBST, the membrane was incubated with horseradish peroxidase-labeled goat anti-mouse (1:5000; Bioss, China) antisera at room temperature for $2 \mathrm{~h}$. Immunoreactive protein bands were visualized with Western Bright ECL (Advansta, USA) reagents following three times wash with TBST.

\section{Results}

In order to characterize mammalian CCPs biochemically and structurally, large quantities of soluble target proteins were required. We found that mouse original CCP6 existed largely as inclusion bodies when expressed in prokaryotic cells. To solve this problem, we optimized the expression conditions, including optimizing the induction temperature and using different receptive cells (Figure 2). Firstly, 2 truncated variants of CCP6 were cloned into pET 28a-HT vector, resulting the plasmids of HT-CCP6-45 and HT-CCP6-167 with a His-tag and a TEV cleavage sites at N-terminal to the sequence of target proteins. Then the plasmids of HT-CCP6-45 and HT-CCP6-167 were transformed into Rosetta (DE3) competent cells, respectively. This strain is a derivative of chloramphenicol resistant plasmid of BL21, supplementation of tRNA corresponding to six rare codons (AUA, AGG, AGA, CUA, CCC, and GGA) that Escherichia coli lacks. In the prokaryotic system, Rosetta (DE3) can improve the expression level of exogenous genes, especially eukaryotic genes. To obtain more target protein expressed in the supernatant, the induction temperature was optimized. Based on the sequences of cloned inserts, the theoretical molecular weights of HT-CCP6-45 is $46 \mathrm{kDa}$, and the theoretical molecular weight of HT-CCP6-167 is $32 \mathrm{kDa}$. The results of coomassie bright blue following SDS-PAGE shown that there is a major band in the precipitate rather than in the supernatant, suggesting that the target protein mainly existed in the form of inclusion bodies (Figure 2A, 2C).

Next, the plasmids of HT-CCP6-45 and HT-CCP6167 were transformed into Arctic Express (DE3) competent cells, respectively. Arctic Express (DE3) contain chaperone GroEL and GroES, which can help the recombinant proteins to fold correctly, reduce the formation of inclusion bodies, and increase the expression and biological activity of soluble recombinant proteins. For HT-CCP6-45, when expressed at $16^{\circ} \mathrm{C}$ with IPTG, the expressed target protein was in the supernatant, while most of the target protein still appeared in the precipitation (Figure 2B). For HT-CCP6-167, when expressed in Arctic Express (DE3), almost all of the target protein appeared in the precipitation (Figure 2D). 
A

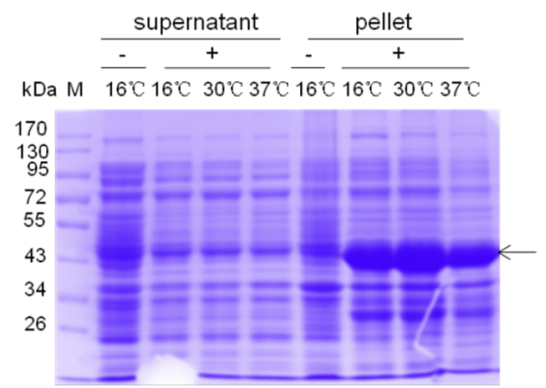

B

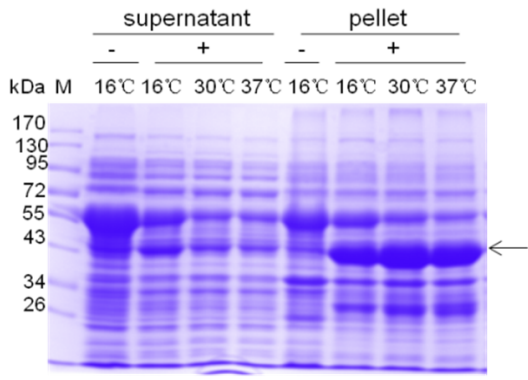

C
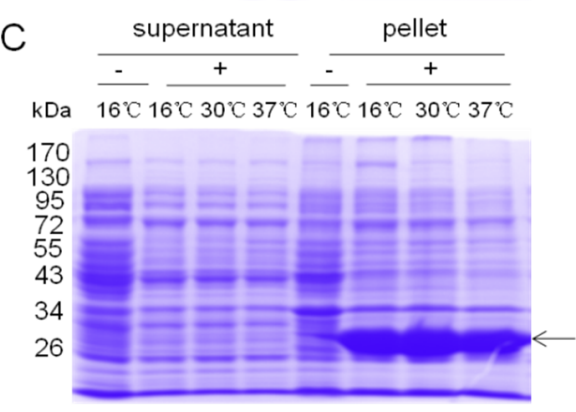

D

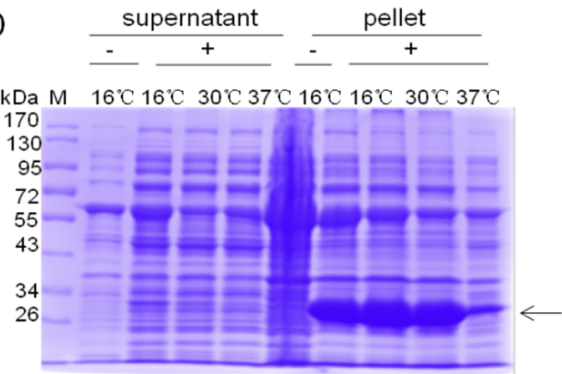

Fig. 2. Protein expression of HT-CCP6-45 and HT-CCP6-167 in different host cells detected by $\mathrm{CBB}$ staining. Protein expressing in bacteria were induced at $16^{\circ} \mathrm{C}, 30^{\circ} \mathrm{C}$ and $37^{\circ} \mathrm{C}$ with or without $0.4 \mathrm{mM}$ IPTG for $18 \mathrm{~h}, 6 \mathrm{~h}$ and $4 \mathrm{~h}$ respectively. The supernatant and precipitation of cell lysates were fractionated by centrifugation and proteins were separated by $10 \%$ SDS-PAGE (A) Protein expression level of HT-CCP6-45 in Rosetta (DE3) showed that the majority of the target protein was in the pellet. (B) Protein expression level of HT-CCP-45 in Arctic Express (DE3) showed that a small amount of target protein was in the supernatant. (C, D) Protein expression level of HT-CCP6-167 in Rosetta (DE3) (C) and Arctic Express (DE3) (D) showed that almost all of the target protein was in the pellet. Arrows indicated positions of the target protein. M: marker.

We found that when plasmid of HT-CCP6-45 transformed to Arctic Express (DE3) competent cell and induced overnight with $0.4 \mathrm{mM}$ IPTG at $16^{\circ} \mathrm{C}$, soluble target proteins could be obtained. Then, the predicted size of HT-CCP6-45 was confirmed by immunoreactive with anti-His antibody (Figure 3). Western blotting of HT-CCP6-45 also detected a band of anti-His immunoreactivity at $\sim 46 \mathrm{kDa}$ (Figure 3 ).

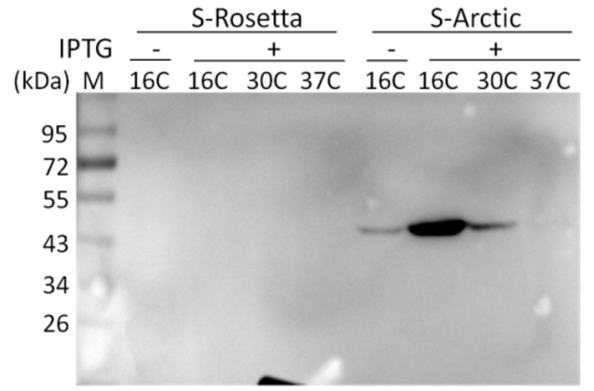

Fig. 3. Protein expression of HT-CCP6-45 in Rosetta (DE3) and Arctic Express (DE3) competent cell, respectively. Protein expressing in bacteria were induced at $16^{\circ} \mathrm{C}, 30^{\circ} \mathrm{C}$ and $37^{\circ} \mathrm{C}$ with or without $0.4 \mathrm{mM}$ IPTG for $18 \mathrm{~h}, 6 \mathrm{~h}$ and $4 \mathrm{~h}$ respectively. Protein expression level of HT-CCP6-45 were confirmed by western blotting using anti-His antibody. S: supernatant; M: marker.

\section{Conclusion}

When mouse CCP6 was expressed in the prokaryotic system, it mainly exists in the form of insoluble fractions in bacterial cells pellet. This is consistent with the recurrent difficulty of getting sufficient quantities of soluble functional domains of human CCPs ${ }^{[12]}$. In order to solve this problem, the expression conditions were carefully optimized. Finally, when the target genes were cloned into pET 28a-HT vector and transformed into Arctic Express (DE3) competent cells (which contain molecular chaperonin, GroEL and GroES that are helpful for proper protein folding), a small amount of soluble target proteins were obtained. In order to achieve a large number of soluble target proteins, the expression conditions still need to be further optimized.

\section{References}

1. Rogowski, K.; Van Dijk, J.; Magiera, M. M.; Bosc, C.; Deloulme, J.-C.; Bosson, A.; Peris, L.; Gold, N. D.; Lacroix, B.; Grau, M. B. Cell 143, 564-578 (2010)

2. Janke, C.; Rogowski, K.; Van Dijk, J. EMBO. Rep 9, 636-641 (2008)

3. Van Dijk, J.; Rogowski, K.; Miro, J.; Lacroix, B.; Eddé, B.; Janke, C. Mol.Cell 26, 437-448 (2007)

4. Wu, H.Y.; Wang, T.; Li, L.; Correia, K.; Morgan, J. I. FASEB. J 26, 4468-4480 (2012)

5. Tort, O.; Tanco, S.; Rocha, C.; Bièche, I.; Seixas, C.; Bosc, C.; Andrieux, A.; Moutin, M.-J.; Avilés, F. X.; Lorenzo, J. Mol. Biol. Cell 25, 3017-3027 (2014)

6. Wu, H.Y.; Rong, Y.; Correia, K.; Min, J.; Morgan, J. I. J. Biol. Chem 290, 1222-1232 (2015)

7. Edde, B.; Rossier, J.; Le Caer, J.-P.; Desbruyeres, E.; Gros, F.; Denoulet, P. Science 247, 83-85 (1990)

8. Rüdiger, M.; Plessman, U.; Klöppel, K.-D.; Wehland, J.; Weber, K. FEBS. Lett 308, 101-105 (1992)

9. Regnard, C.; Desbruyères, E.; Huet, J.-C.; Beauvallet, C.; Pernollet, J.-C.; Eddé, B. J. Biol. Chem 275, 
15969-15976 (2000)

10. Van Dijk, J.; Miro, J.; Strub, J.-M.; Lacroix, B.; Van Dorsselaer, A.; Edde, B.; Janke, C. J. Biol. Chem 283, 3915-3922 (2008)

11. Rodríguez de la Vega Otazo, M.; Lorenzo, J.; Tort, O.;
Avilés, F. X.; Bautista, J. M. FASEB. J 27, 424-431 (2013)

12. De La Vega, M. R.; Sevilla, R. G.; Hermoso, A.; Lorenzo, J.; Tanco, S.; Diez, A.; Fricker, L. D. FASEB. J 21, 851-865 (2007) 